\title{
TEMPERATURE, ABUNDANCE AND MAGNETIC MAPPING OF STELLAR ATMOSPHERES
}

\section{J.-F. DONATI}

University of Western Ontario, London Ontario, N6A 3K7 Canada

\begin{abstract}
In this paper, I review recent progress in the domain of indirect imaging of stellar atmospheres. Since all stars other than the Sun are too far away for us to disclose any feature on their surface directly, one has to use an indirect tool to image their atmosphere. This method (Doppler Imaging) takes advantage of a star's rotation to identify and characterise inhomogeneities on its surface. This is done by studying both how inhomogeneities (carried in and out of the observer's view by rotation) modulate photometric and spectroscopic data with time, and how surface features distort the shape of line profiles at a given rotational phase. In the last decade, Doppler Imaging has first been applied to abundance mapping of magnetic Ap stars, then extended to temperature and magnetic mapping of cool active stars. Allowing a solar-like way of studying the surface of stars, this technique provides a wealth of information about stellar atmospheres and, in particular, about the physical processes which dictate their dynamics and energy balance.
\end{abstract}

\section{INTRODUCTION}

In ancient Greece and China, astronomers first observed that the surface of our Sun was non uniformly bright, and covered with dark spots. Since then, we have learned a lot about the inhomogeneous and stratified aspect of the solar atmosphere; for instance, kG magnetic fields have been found in spots, granules are observed in the quiet photosphere, plages are seen above spots at the chromospheric level. In particular, we know now that, despite their small relative size, these features are extremely important and witness the interaction between dynamical and radiative processes in the solar atmosphere, as well as their structuration by the magnetic field. Therefore, in the same way as the interpretation of some physical phenomenon at a macroscopic scale may require a microscopic description, the understanding of the overall physics of the Sun's atmosphere calls for unravelling the processes at work in small size inhomogeneities.

Most information we have already and we expect to get soon about fine solar atmospheric structures comes from angularly resolved observations, allowed by the proximity of the Sun relative to the Earth. Among stars other than the Sun, the M2 supergiant star Betelgeuse ( $\alpha$ Ori) is the only one for which a recent interferometry study (Buscher et al. 1990) concludes that a large scale bright feature is probably present on its visible hemisphere. However, due to the resolution limit of the method, no fine detail of the stellar surface is revealed, 
though this star has the largest angular diameter after the Sun. For all other stars, we cannot yet investigate details on their surfaces with direct imaging methods and will likely not be able to do so in a close future. However, for some type of stars and in particular for magnetic Ap and cool active stars, we already have ample evidence, through photometric and spectroscopic observations, that their surface is highly non uniform.

This inhomogeneous aspect of stellar surfaces was first suggested by the fact that certain indexes (like the equivalent width of given spectral lines, the discintegrated longitudinal component of the magnetic field or UBVRI luminosities of the star) are showing periodic modulation. Since different types of indexes in a given star are varying with the same frequency, the observed modulation was attributed to the star's rotation, carrying features successively in and out of the observer's view (Stibbs 1950; Kron 1952). For rapid rotators, more information about the non uniform surface distribution at a given rotational phase may be found in the often asymmetric shape of rotationally broadened spectral lines; each profile can indeed be seen as a one-dimensional map of the star's visible disc, resolved by Doppler effect in a direction perpendicular to both rotation axis and line of sight, but blurred in the orthogonal direction (Pyper 1969; Fekel 1983).

Both time modulation and line profile distortions of stellar spectra have been used since early attempts to map surfaces of magnetic Ap stars (Pyper 1969) or solar-type stars (Dorren et al. 1981; Vogt \& Penrod 1983). In cool active stars, light curves and profile distortions are attributed, as in the Sun, to temperature (i.e. brightness) spots unevenly distributed over the stellar surface and probably caused by the emergence of magnetic fields out of the photosphere. On magnetic Ap stars, by contrast, it is now widely accepted that chemical abundance rather than temperature varies from one point of the atmosphere to another, in relation with the surface magnetic field. In both cases, mapping techniques aim at recovering an image of the atmosphere from the observations. Computing light curves or line profiles produced by a rotating star with a known atmospheric model, geometry and distribution, is a relatively straightforward task. The problem at hand is the inverse of this.

The very first method was developed for magnetic Ap stars (Deutsch 1970) and consisted in a direct analytical inversion. From the observed equivalent width and mean Doppler shift periodic variations of a disc-integrated line, one could, in principle, determine the coefficients of a spherical harmonics expansion of the distribution of local equivalent widths at the surface of the star. However, for two main reasons, this technique turned out to be ineffective. Since the expansion series was truncated after a few terms to keep the analytical problem tractable, the type of surface feature that could be imaged was severely restricted. Moreover, only the integral characteristics of the line profiles were used, while the very rich information contained in their shape was neglected. Although several improvements were brought to this first method to take better account of the profile shape of lines (e.g. Pyper 1969; Falk \& Wehlau 1974, Mégessier 1975), it was finally abandoned and replaced by a new generation of more powerful tools.

Instead of a direct inversion, several groups began trying to image stars by manual trial and error iterative procedures (Khokhlova 1975; Vogt \& Penrod 1983). At the same time, pioneering work by Goncharskij et al. (1982) 
in attempting to define stellar imaging more rigorously led to the understanding that this problem is fundamentally ill-posed, i.e. has multiple solutions. An additional criterion must be invoked to select one of them, for instance the "simplest". Recently, robust and efficient algorithms have been developed to make the whole imaging process (Doppler Imaging) fully automatic. Extensive simulations were also carried to determine when test distributions are correctly reconstructed, i.e. what sort of data requirements are needed to recover successfully an input image (Vogt et al. 1987; Rice et al. 1989; Piskunov et al. 1990; Brown et al. 1991).

In this paper, I first outline the principles of Doppler Imaging and point out the individual peculiarities of temperature, abundance and magnetic mapping. I then describe the important results that have been obtained to date in this domain, and their implications for our understanding of stellar atmospheres. To conclude, I mention which orientations stellar imaging could take soon, as well as the possible astrophysical fallouts.

\section{IMAGING METHOD}

\section{Principles}

As mentioned in the introduction, the profile shape of spectral lines is the richest source of information about non uniform surface distributions on a rapidly rotating star. Due to Doppler effect induced by rotation, the visible disc of the star is sliced into strips of constant Doppler shift. Each inhomogeneity on the disc will then affect in some way the spectral contribution of the corresponding isovelocity strip, and thus distort the disc-integrated profile of the line at this given Doppler shift. Hence, each profile can be seen as a 1D distribution of the visible hemisphere of the star. From a time series of similar 1D distributions, one can follow how widely and how fast these distortions move across the line profile during a rotational cycle, and infer some accurate information about the location and shape of the parent inhomogeneities, as well as about their physical properties. Using profile sets in all Stokes parameters (intensity $I$, linear polarisations $Q$ and $U$, circular polarisation $V$ ) and for various spectral lines should, in principle, allow for the simultaneous reconstruction of abundance/brightness and magnetic distributions.

Formally, the relation between the image $I$ to be reconstructed and the observed data set $\mathbf{D}$ may be written $\mathcal{R}(\mathbf{I})=\mathbf{D}$ where $\mathcal{R}$ notes a function which includes all line formation calculations required when computing profiles from a known atmospheric distribution I, model structure and geometry. Since the problem is ill-posed (Goncharskij et al. 1982), a regularisation function $S$ is used to select one of the solutions compatible with the data, for instance the "simplest" or "smoothest" one. For that purpose, the Entropy function (Skilling \& Bryan 1984) and the Tikhonov function (Tikhonov 1963) have been invoked most of the time, both yielding very similar results (Piskunov 1991). The inversion hence resumes to maximise a Lagrange function $Q=S-\lambda C(\lambda$ is a conventional Lagrange multiplier), i.e. to maximise $S$ under the constraint of a fit to the data at an accuracy of $C=C_{a i m}$ where $C$ is a statistic (for instance a chi-square) and $C_{a i m}$ the desired accuracy of the fit. An iterative algorithm (often based on a conjugate gradient method) is used to converge towards the 
solution.

To compute the function $\mathcal{R}$ and its gradient at each iteration, LTE line formation routines with atmospheric models are used. Since local profiles need to be obtained at each grid cell on the stellar surface, at each rotational phase and at each iteration of the code, direct integration of the transfer equation as part of the imaging process requires an enormous amount of computing time, that only super computers can handle. To overcome this problem, tabulated LTE profiles corresponding to different abundances, temperatures and limb angles are first built prior to the imaging process. Then, during the inversion, the code interpolates in this table to obtain the profiles needed. Though sophisticated already, intrinsic profiles calculations could be wrong by a significant amount, due for instance to the unrealistic LTE atmospheric models used. However, in these rapidly rotating stars, the rotational profile is usually much wider than the intrinsic one, whose main effect is thus line broadening. The synthetic shape of local profiles has therefore little influence on the imaging process.

Of course, Doppler Imaging relies on several assumptions. It assumes first that the observed profile variations are entirely due to rotational modulation. If the observed profile variations are correctly reproduced by the imaging process and sufficient phase coverage is obtained, it usually implies that this assumption is verified a posteriori (e.g. Hatzes 1990). This can also be checked independently by comparing profiles recorded at a given phase, but from different rotational cycles. In addition, Doppler Imaging often assumes that the star is spherical and rotates as a rigid body, but could easily cope with ellipsoidal stars and differential rotation if needed. However, these effects are likely to be quite small on the inferred maps.

In case of a slow rotator $(v \sin i<10 \mathrm{~km} / \mathrm{s}$ where $v$ notes the star's equatorial velocity and $i$ the inclination angle of its rotational axis to the line of sight) or poor spectral resolution [instrumental profile with $H W H M(\mathrm{~km} / \mathrm{s})>v \sin i$ ], spectral profiles can no longer be seen as 1D distribution of the star, since they mainly reflect a local profile averaged over the disc and smeared by the instrumental broadening. Only disc-integrated quantities, such as BV luminosity, line equivalent width, average longitudinal magnetic field, can then be measured throughout the rotational cycle. If such variations can be accounted for with simple spotted (e.g. Dorren et al. 1981) or off-centred dipole (Borra \& Landstreet 1980) structures, this sort of data modelling has to assume a priori a given kind of structure to be imaged (e.g. one with two cool circular spots of variable size and location). Since such models can be totally wrong [e.g. starspots could be bright rather than dark (Vogt 1981; Donati et al. 1992b)] and, at the same time, perfectly account for the observed data, the derived results highly depend on the assumed structure rather than just on the observations. For this reason, such modelling processes are not considered as true imaging and will not be discussed herein. However, some of the results obtained will be mentioned.

Before working with real data, it is important to check with adequate simulations that imaging behaves well in the particular case one plans to use it, and to test for instance that the data to be inverted contain enough spatial information to characterise the parent structure. A basic simulation consists in two steps. A synthetic image is first produced, from which sets of profiles are computed and artificially noised. In a second step, a map is recovered from these profiles and compared to the original one. Simulations with different kinds 
of synthetic maps have been presented to date in the literature. Most of them describe reconstructions of sharp-edged features such as spots, deviating from a uniform background and usually covering a small fraction of the total stellar surface. Some others simulate reconstructions of structures smoothly varying from one surface point to another and covering most of the stellar surface, such as dipoles. In the following sections, I review the major results obtained from simulations with these two different kinds of images, referred to as "spotted" and "dipolar-like" distributions.

\section{Temperature and abundance mapping}

On a cool star, a dark spot causes a deficit of photons to the isovelocity strip in which it is located, therefore decreasing its intensity-weighted area. Since the intensity-weighted area of an isovelocity strip is proportional, at first order, to the depth of a disc-integrated profile at the corresponding Doppler shift, it implies that a dark spot shows up as a hump in a disc-integrated profile. For the strong lines commonly used in cool stars imaging, line equivalent width variations with temperature have usually smaller effects than continuum variations and can be seen as second order perturbations. On magnetic Ap stars by contrast, the temperature is roughly constant over the photosphere and local abundance inhomogeneities generate local increase or decrease in line equivalent widths, producing respectively notches or humps in disc-integrated profiles.

Several papers (Vogt et al. 1987; Rice et al. 1989; Hatzes et al. 1989; Piskunov et al. 1990; Piskunov \& Wehlau 1990; Piskunov 1991; Brown et al. 1991, Jankov \& Foing 1992) present simulated imaging of synthetic brightness and abundance maps. For both brightness and abundance imaging, spotted distributions are found to be reasonably reconstructed; in particular, both shape and location of spots are well recovered, while the brightness or abundance deviation from the uniform background is sometimes slightly underestimated. If brightness imaging of dipolar-type structures behaves quite well (Brown et al. 1991), abundance distributions of similar type are usually less accurately recovered, with poles being dragged slightly but significantly to the subobserver latitude.

The influence of low $S / N$, low resolution or badly phase-sampled data have been discussed extensively in the papers quoted above, the main consequence being a progressive lessening and smearing of details in the reconstructed map. More problematic are errors resulting from a wrong guess of some atmospheric parameters like $v \sin i, i$ or the limb darkening constant. These may cause spurious features in the derived images (Vogt et al. 1987). Such artifacts are easy to detect though and are actually used for a fine tuning of atmospheric parameters.

The strongest limitation in Doppler Imaging is that the profile corresponding to a uniform photosphere, from which distortions are measured and interpreted, is basically unknown. Indeed, this "undisturbed photospheric profile" is scarcely observed, since such stars often show at least one spot at each rotational phase. We thus have to take the classical round-shape rotational profile as granted. If this round-shape profile correctly accounts for lines of unspotted rapid rotators (such as Am stars), it could be affected in spotted stars by, say, the presence of a chromosphere. Since such perturbations tend to distort the profiles at all different phases the same way, latitude independent features (i.e. 
rings) encircling the star and polar artifacts are expected. For this reason, it is safer to confirm each feature reconstructed by Doppler Imaging, whenever independent results, such as magnetic maps (see below), are available.

In a general case, temperature/abundance and magnetic mappings should be done simultaneously. Indeed, magnetic fields can broaden significantly and even split Stokes $I$ profiles of spectral lines. For fields of several kG, such distortions are comparable in size to those produced by temperature/abundance inhomogeneities. However, in the case of surface fields weaker than about $1 \mathrm{kG}$, unpolarised profiles are only slightly affected and temperature/abundance mapping can be dissociated from magnetic imaging (Rice \& Wehlau 1990).

\section{Magnetic mapping}

To study stellar magnetic fields, we usually analyse Zeeman distortions of spectral lines. Since Zeeman effect is, by far, the likeliest cause of circular (Stokes $V$ ) or linear (Stokes $Q$ and $U$ ) polarisation in line profiles, observing in polarised light is considered as the optimal tool for investigating stellar magnetic structures. In this kind of study, circular polarisation signatures are more often measured than linear polarisation profiles, which are usually about an order of magnitude smaller and thus only accessible for stars with fields of at least several kG. In case of very strong fields, Stokes $I$ profiles may exhibit Zeeman splitting if rotational broadening is small (i.e. $v \sin i<20 \mathrm{~km} / \mathrm{s}$ ). For most stars rotating rapidly enough to be Doppler imaged, Zeeman signatures in unpolarised profiles mainly reduce to line broadening, which can be identified reliably only for fields stronger than several $\mathrm{kG}$.

Magnetic mapping from polarisation profiles of spectral lines (ZeemanDoppler Imaging or ZDI) was first proposed by Semel (1989) and studied more extensively in following papers (Donati et al. 1989; Donati \& Semel 1990; Donati 1990; Brown et al 1991; Semel et al. 1992). It has several advantages compared to conventional Doppler Imaging. As magnetic field is the likeliest source of polarisation in line profiles, the "undisturbed photospheric profile" involved in the imaging process is now perfectly known; for a null field structure, Stokes $Q, U$ and $V$ profiles are indeed flat by definition. No spurious features, and in particular, no polar artifacts can be generated in magnetic images, which are thereby much more reliable than temperature or abundance maps. Such images can be used to check independently the reality of brightness/abundance features reconstructed in conventional Doppler images.

However, ZDI has certain limitations as well. Brown et al. (1991) showed that only spotted magnetic structures (Fig. I) can be reconstructed from sets of circular polarisation Stokes $V$ profiles. If the information coming from sets of linear polarisation profiles is not incorporated, dipolar-like structures are not correctly recovered. In particular, the poles of an originally dipole field distribution are both reconstructed at the subobserver latitude; hence, distributions "simpler" than the original one are compatible with the data. This means essentially that sets of Stokes $V$ profiles do not contain enough spatial information about magnetic dipolar structures, making them impossible to be recovered unambiguously when sets of Stokes $Q$ and $U$ profiles are not used.

Another drawback is the fact that fields in very dark or under-abundant regions may easily be dimmed or even totally obscured since the polarisation 

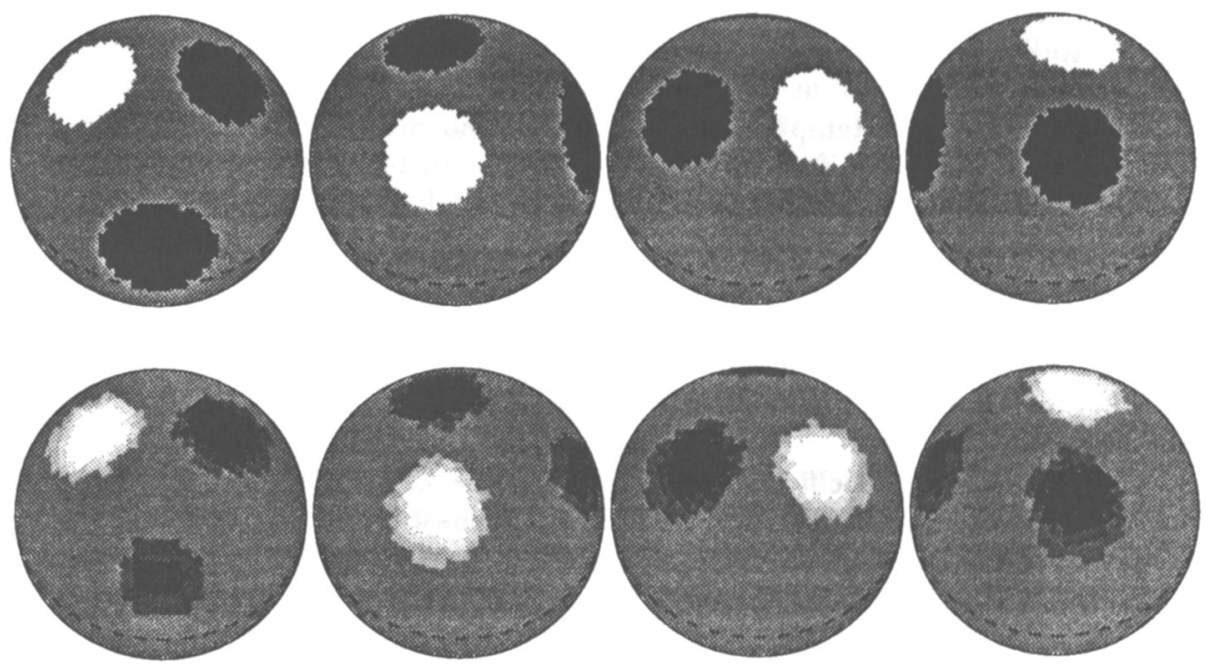

FIGURE I Maximum entropy reconstruction (lower row) of a synthetic spotted magnetic distribution (upper row) from Stokes $V$ data. From left to right, the star is shown at rotational phases $0.00,0.25,0.50$ and 0.75 (as in all following figures). The location, field intensity, and shape of spots are reasonably well recovered (Brown et al. 1991).

rate generated by such features is very small, and partially hidden by photon noise. Using a brightness or abundance image derived from corresponding Stokes $I$ data sets may partly compensate for this problem, but cannot, of course, regenerate the missing information drowned by noise.

\section{RESULTS}

\section{Magnetic Ap stars}

In magnetic Ap stars, elements are spread inhomogeneously over the stellar surface, in a distribution which often reflects the magnetic structure. According to the diffusion theory (Michaud 1970), the strong fields observed on such stars inhibited large scale mixing processes like convection, meridional circulation and turbulence in the outer layers of the star, leading to a very stable photosphere. Microscopic diffusion then becomes the dominant transport process at the stellar surface, with neutral elements migrating radially while ions follow the field lines. However, this so called "simple diffusion model" can only explain the average abundance anomalies observed for instance on 53 Cam (Landstreet 1988), but fail to account for the abundance variations over the surface of this star (Babel $\&$ Michaud 1991a). The effects of magnetic fields on abundance distributions (Vauclair at al. 1979; Michaud et al. 1981; Mégessier 1984; Babel \& Michaud $1991 \mathrm{~b})$ is not well understood yet. In particular, inhomogeneous mass loss seems required to match observations better. What have we learned to date from surface imaging of magnetic Ap stars? 
Fields of magnetic Ap stars have been extensively studied since Babcock (1958) detected them. Till recently, works on fields of magnetic Ap stars mainly consisted in measuring the disc-averaged longitudinal and surface field throughout the rotational cycle of a star and fitting them with centred or off-centred dipole models (e.g. Borra \& Landstreet 1980; Mathys 1991) or more complex non axisymmetric models (Stift \& Goosens 1991). Very few studies measured polarisation profiles of spectral lines and used their shape to derive the parameters of an assumed dipolar magnetic distribution (Borra 1980; Piskunov 1985; Glagolevskij et al. 1985; Donati et al. 1990b). Though such fits with dipole models are not true imaging methods (since they assume a priori a given large scale topology of the field), they still remain the best way to characterise magnetic structures of Ap stars till magnetic maps of dipolar-like distributions can be recovered. This should be possible, in principle, when linear polarisation profiles of spectral lines can be measured (Brown et al. 1991).

In stars with strong magnetic fields, Stokes $I$ profiles show clear Zeeman distortions. Since magnetic fields cannot be mapped accurately yet, abundance mapping is also subject to caution; hence, only rough information about the abundance distribution of elements may be inferred (e.g. Landstreet 1988). However, even such limited spatial information proved to be very useful to constrain better diffusion models, and to show that mass loss seems required to explain the geographic distribution of elements on the surface of 53 Cam (Babel \& Michaud 1991a; Babel 1992).

In the case of weak field Ap stars (surface field $<1 \mathrm{kG}$ ), Stokes $I$ profiles are predominantly distorted by abundance inhomogeneities so that Doppler Imaging becomes valid. Within the last three years, considerable effort has been realised in trying to recover accurate abundance maps of magnetic Ap stars, and images have been derived for 6 different objects, mainly from elements like $\mathrm{Fe}, \mathrm{Cr}$ and Si (Hatzes et al. 1989; Rice \& Wehlau 1990, 1991; Hatzes 1990; Donati 1990; Hatzes 1991a, 1991b). There is some general agreement on some typical features found in these maps. All images show evidence of rings and spots distributed in a basically axisymmetric distribution. The observed rings are often irregular (e.g. Rice \& Wehlau 1991). For a given star, distributions in different elements have the same axis of symmetry (Hatzes 1991a). Note that, for stars on which a field was detected, the symmetry axis of the abundance distribution sometimes differ from the magnetic axis derived from dipole modelling (e.g. Rice \& Wehlau 1991). However, the derived abundance and magnetic poles, whenever non coincident, always agree in longitude. The remaining discrepancy is likely at tributable to the large latitude uncertainties in the process of dipole modelling of disc-integrated magnetic data (such as $\mathrm{H} \beta$ polarimetric measurements).

Inhomogeneous element distributions in such stars are therefore very likely induced by the magnetic structure, as first proposed by Stibbs (1950). In such a context, the Si-depleted spots and Si-enhanced irregular rings observed on $\theta$ Aur (Rice \& Wehlau 1991; Hatzes 1991a), $\gamma^{2}$ Ari (Hatzes et al. 1989) and BP Boo (Hatzes 1990) could indicate respectively the magnetic poles and equator. By contrast, on $\epsilon$ UMa (Rice \& Wehlau 1990; Hatzes 1991b), $\theta$ Aur (Rice \& Wehlau 1990; Hatzes et al. 1991a), $\omega$ Her and 45 Her (Hatzes 1991b), Cr seems depleted at the magnetic equator and at one pole at least, while intermediate regions are over-abundant. Fe is sometimes depleted at the magnetic poles and enhanced at the magnetic equator as in $\alpha^{2}$ CVn (Khokhlova \& Pavlova 1984), or vice versa 
as in $\epsilon$ UMa (Donati 1990; Rice \& Wehlau 1991). Little has been done to date about rare-earth abundance distributions in magnetic Ap stars. Early results on $\alpha^{2}$ CVn (Goncharskij et al. 1983) suggest that these elements concentrate near one or both magnetic poles.

\section{Solar-type stars}

Dynamo models describe tentatively the MHD processes observed at the surface of the Sun and other cool active stars. A weak radial/poloidal magnetic field is first generated by rotation in the convection zone of a cool star. Differential rotation then winds field lines in a mainly toroidal structure. Cyclonic motions in the convective zone act to bring back the initial radial/poloidal magnetic structure through reconnection of field lines (Parker 1970). As this process goes on, many activity demonstrations are observed, such as photospheric sunspots witnessing the emergence of flux tubes out of the convective zone by buoyancy, or strong emission lines produced by a high-temperature chromosphere and transition region. If observations have shown us already that such phenomena also occur on many other active stars, only stellar imaging can bring accurate information about their local physical properties.

However, most cool dwarfs, subgiants or giants of spectral type later than early G rotate very slowly $(v \sin i<10 \mathrm{~km} / \mathrm{s}$ ) due to some kind of magnetic breaking or/and angular momentum redistribution process starting at about this evolutionary stage. As a result, indirect surface imaging as described above does not apply for such slow rotators since the spatial information contained in the profile shape of spectral lines is greatly reduced. In this case, rotational modulation (e.g. light curves) yields most of what we can learn about temperature distributions of such stars (e.g. Vogt 1981; Rodonò et al. 1986). Although the simple spot modelling often used for such data is not true imaging, it can still be very informative when analysing long term variability, like changes in light curves shape (e.g. Strassmeier \& Bopp 1992); such a method indeed evidenced that differential rotation in cool active stars decreases when rotation rate increases (Hall 1991). In addition to rotational modulation, one can also use the very small variability in profile shape by looking at changes in line bisectors (Toner \& Gray 1988). If this can bring further information and support on the location and physical properties of surface inhomogeneities, the somewhat arbitrary spot model is still required.

Even if less numerous, rapidly rotating cool active stars can yield very important clues about activity phenomena, observed to strengthen with increasing rotation rates (Strassmeier at al. 1990; Saar 1990). Most rapid rotators in this spectral class are associated in close binary systems, whose orbital angular momentum sustains, through tidal coupling ( $\mathrm{Zahn}$ 1977), a high rotation rate for both components. For 6 objects, most of them being RS CVn systems, temperature images have been reconstructed to date (Vogt 1988; Piskunov et al. 1990; Strassmeier et al. 1991; Vogt \& Hatzes 1991; Donati et al. 1992b; Strassmeier et al. 1992). Recently, ZDI has been applied to RS CVn binaries (Donati et al. $1990 \mathrm{a}$ ), leading to the detection of circularly polarised Zeeman signatures in 4 systems (Donati et al. 1992a), and to the first magnetic mapping of a solar-type star other than the Sun (Donati et al. 1992b).

Although discrepancies still exist between images reconstructed by different 

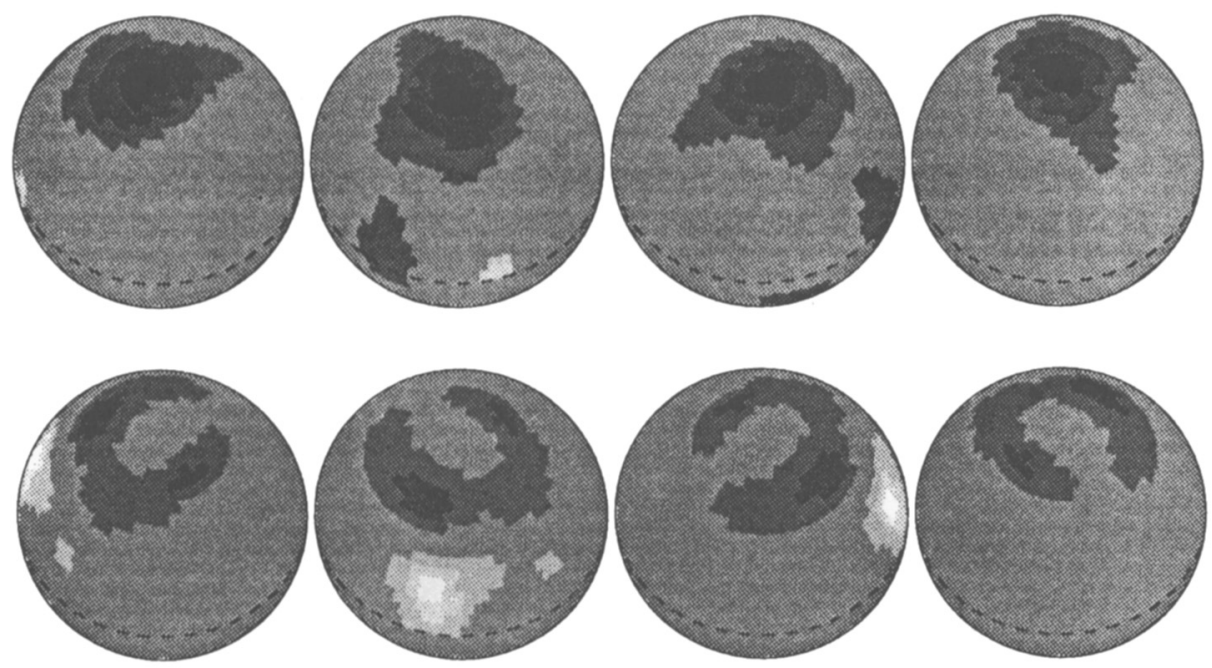

FIGURE II Temperature (upper row) and magnetic (lower row) photospheric image of the K1 subgiant of HR 1099 obtained with Zeeman-Doppler Imaging (Donati et al. 1992b). Magnetic regions are found to be associated with toroidal field (indicated by darker and brighter grey levels with steps of $200 \mathrm{G}$ ) and to coincide with cool spots in the temperature image.

groups from the same data set (Strassmeier et al. 1991), some results seem relatively well established now. Large starspots, about $1000 \mathrm{~K}$ cooler than the photosphere, often develop at high latitudes (and sometimes even on the pole) in such objects, while sunspots are confined to equatorial belts. Since Doppler Imaging can produce polar artifacts, the concept of polar spot has been very controversial over the last few years. However, new temperature and magnetic images obtained for the K1 subgiant of the RS CVn system HR 1099 by Donati et al. (1992b) suggest that magnetic fields of several hundred $G$ are associated with polar spots, thereby strongly supporting the reality of such features. Apparently, polar spots are long-lived and bear some resemblance with solar coronal holes, as noted by Vogt (1988); being associated with fields at least 100 times stronger than solar, these features could become prominent at the photospheric level in these stars, while they are only visible at the coronal level in the Sun. Both bright and dark equatorial features are also detected on these stars (e.g. Piskunov et al. 1990), though often smaller and more transient than polar ones.

The magnetic image of HR 1099 (Donati et al. 1992b) shows that starspots are not active solar complexes made of many small spots of opposite polarities [as proposed earlier by Vogt (1988)], but large unipolar magnetic regions (see Fig. II). Moreover, the field orientation in these regions varies with time; Stokes $V$ spectra of HR 1099 suggest that the magnetic structure of the K1 subgiant evolved from a radial/poloidal structure at epoch 1989.6 to a mainly toroidal configuration at epoch 1990.9 , probably due to the winding of field lines around the star by differential rotation (Donati et al. 1992b). From a time series of images obtained at nearby epochs, Vogt \& Hatzes (1991) find that differential 
rotation in the rapidly rotating K0 subgiant of UX Ari is significantly smaller than solar [in agreement with the result of Hall (1991)], but with a pole rotating faster than the equator.

\section{FUTURE ORIENTATIONS IN STELLAR IMAGING}

If stellar imaging produced many valuable results already, it is still in its infancy and many other directions shall be explored soon and reveal new clues about diffusion and dynamo processes at work in stellar atmospheres.

Several observational strategies may lead to a better understanding of diffusion mechanisms in magnetic Ap stars. Firstly, for each observed star, it is important to obtain abundance maps from many different elements in both ironpeak and rare-earth groups, having very different sensitivities to diffusion processes. At the same time, true mappings of the magnetic structure of such stars (from a simultaneous inversion of all four Stokes profiles) could tell us how local abundance inhomogeneities relate to variations in both field strength and orientation. Such comprehensive information could help in identifying, modelling and constraining the main process (possibly mass loss) leading to surface inhomogeneities in the atmosphere of such stars (Babel \& Michaud 1991a; Babel \& Michaud 1991c; Babel 1992). Finally, abundance maps at different wavelengths (from UV to IR) could be derived to obtain further observational evidence and accurate measurements of the stratification of elements in the photosphere of magnetic Ap stars (Lanz 1987; Babel \& Lanz 1992). In particular, this should also allow quantitative tests of diffusion theories and models.

Indirect imaging can also bring valuable information about dynamo processes in cool active stars. For instance, one key-project of the coming years could consist in monitoring a few stars throughout their activity cycle, i.e. getting several atmospheric images per year for a total period of about a decade. From such data, one could first study how spots appear, migrate and disappear on the stars' surface. In particular, this will allow measurements of differential rotation which could be used to clarify the role of this parameter in dynamo models. Simultaneous magnetic images will also show how flux tubes emerge from the photosphere, and how the global magnetic structure is progressively distorted during an activity cycle. Particularly interesting should be the changes in field orientation when the star evolves from activity minimum to activity maximum, and then back to activity minimum (Donati et al. 1992b). Finally, indirect imaging could be extended to the upper atmosphere of such stars, as initiated by Gondoin (1986) and Neff et al. (1989), but in a much more sophisticated way. Simulations suggest that chromospheric and transition region maps could be reconstructed (see for instance Fig. III) using UV PRISMA data $(S / N=50$ and $\lambda / \Delta \lambda=30000)$. Images from several lines could yield temperature and density distributions with height in active regions on various stars. In particular, this could bring new clues in our understanding of chromospheric and coronal heating in cool active stars.

As instrumentation improves, other classes of stars could be studied with surface imaging. In particular, investigations of $\mathrm{T}$ Tauri, BY Dra and Ae/Be Herbig stars could bring an unprecedented highlight of atmospheric phenomena in early stages of stellar evolution. 
Fig. IIIa
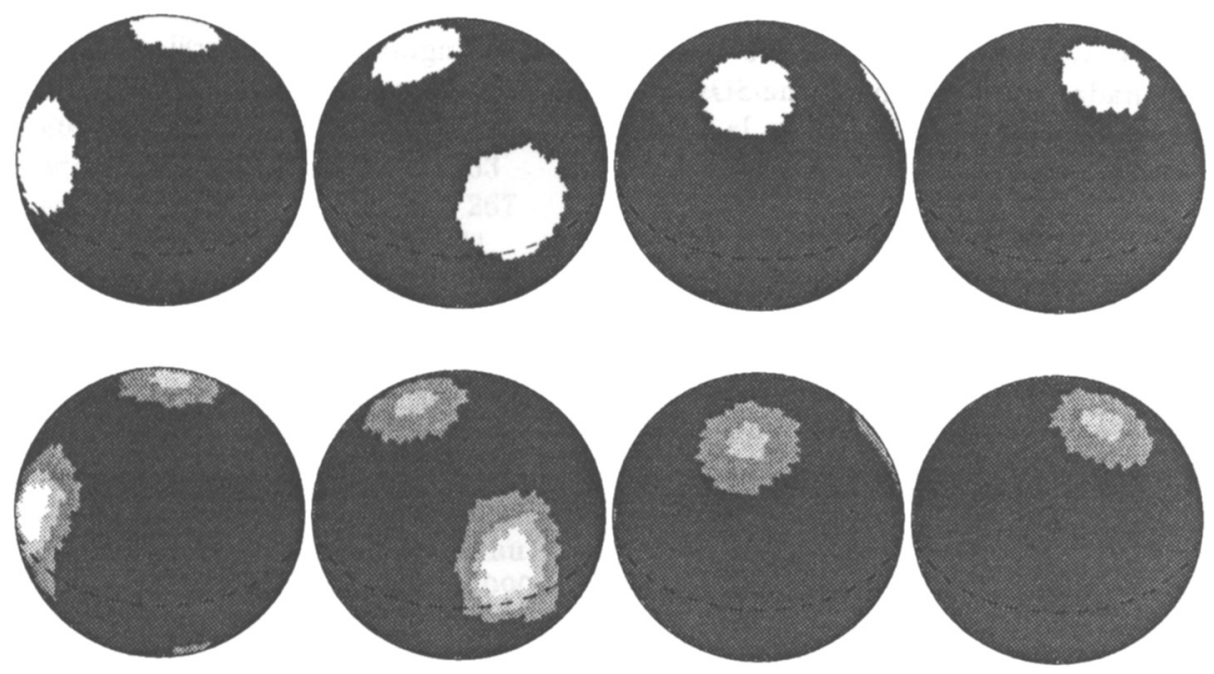

Fig. IIIb

Simulated TR imaging from synthetic $C N$ profiles

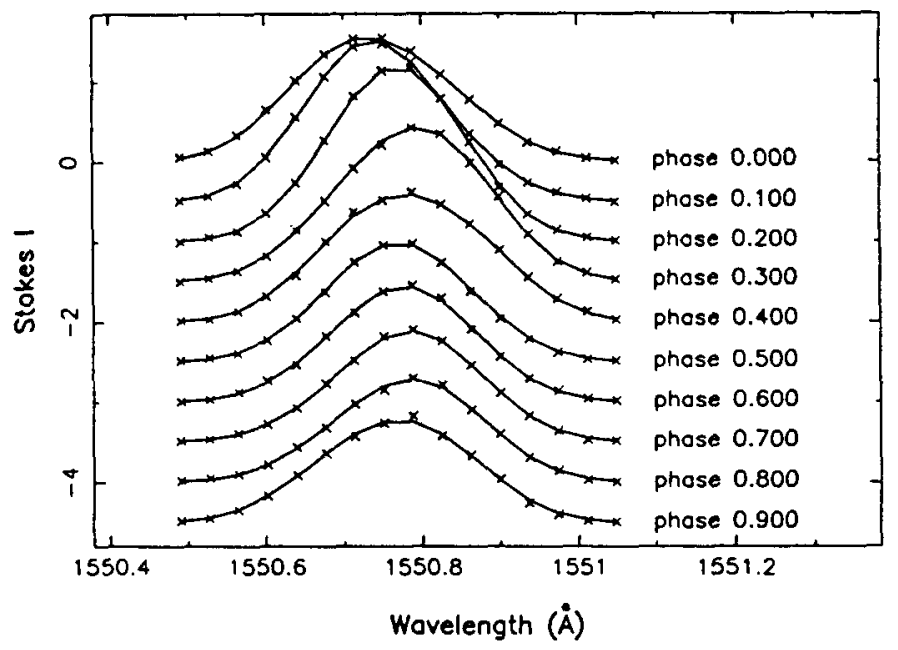

FIGURE III Simulated maximum entropy reconstruction (lower row of a) of a synthetic transition-region spotted structure (upper row of a) from synthetic C IV line profiles. Grey levels indicate line strength with steps of $200 \%$ while medium grey indicates the quiet transition region. In $b$, the synthetic C IV profiles (crosses) used for the maximum entropy fit (full line) correspond to UV data as PRISMA could collect them. 


\section{ACKNOWLEDGMENTS}

I would like to thank F. Praderie for a critical reading of the manuscript. This work was supported by the Natural Science and Engineering Research Council of Canada. I warmly thank the SOC of the IAU Colloquium 138 "Inside the Stars" and the Centre National de la Recherche Scientifique for providing additional travel funds.

\section{REFERENCES}

Babcock H.W., 1958, ApJS 3, 141

Babel J., 1992, A\&A (in press)

Babel J., Lanz T., 1992, A\&A (submitted)

Babel J., Michaud G., 1991a, ApJ 366, 560

Babel J., Michaud G., 1991b, A\& A 241, 493

Babel J., Michaud G., 1991c, A\&A 248, 155

Bopp B.W., Fekel F.C., 1976, AJ 81, 771

Borra E.F., 1980, ApJ 235, 915

Borra E.F., Landstreet J.D., 1980, ApJS 42, 421

Brown S.F., Donati J.-F., Rees D.E., Semel M., 1991, A\&A 250, 463

Buscher D.F., Haniff C.A., Baldwin J.E., Warner P.J., 1990, MNRAS 245, 7P

Deutsch A.J., 1970, ApJ 159, 985

Donati J.-F., 1990, PhD thesis, Université Paris VII

Donati J.-F., Brown S.F., Semel M., Rees D.E., et al., 1992b, A\&A (in press)

Donati J.-F., Semel M., 1990, Solar Phys. 128, 227

Donati J.-F., Semel M., Praderie F., 1989, A\&A 225, 467

Donati J.-F., Semel M., Rees D.E., 1992a, A\&A (in press)

Donati J.F., Semel M., Rees D.E., Taylor K., Robinson R.D., 1990a, A\&A 232, L1

Donati J.-F., Semel M., del Toro Iniesta J.C., 1990b, A\&A 233, L17

Dorren J.D., Siah M.J., Guinan E.F., McCook G.P., 1981, AJ 86, 572

Falk A.E., Wehlau W.H., 1974, ApJ 192, 409

Fekel F.C., 1983, ApJ 268, 274

Glagolevskij Y.V., Piskunov N.E., Khokhlova V.L., 1985, Pis'ma AZh 11, 371 (SvA Lett. 11, 154)

Goncharskij A.V., Stepanov V.V., Khokhlova V.L., Yagola A.G., 1982, AZh 59, 1146 (SvA 26, 690)

Goncharskij A.V., Ryabchikova T.A., Stepanov V.V., Khokhlova V.L., Yagola A.G., 1983, AZh 60, 83 (SvA 27, 49)

Gondoin Ph., 1986, A\&A 160, 73

Hall D.S., 1991, in: The Sun and Cool Stars: Activity, Magnetism, Dynamos. IAU Coll. 130, 353. Tuominen I., Moss D., Rüdiger G. (eds.). Springer, Berlin Heidelberg New York

Hatzes A.P., 1990, MNRAS 245, 56

Hatzes A.P., 1991a, MNRAS 248, 487

Hatzes A.P., 1991b, MNRAS 253, 89

Hatzes A.P., Penrod G.D., Vogt S.S., 1989, ApJ 341, 456

Jankov S., Foing B.H., 1992, A\&A 256, 533 
Khokhlova,V.L., 1975, AZh 52, 950 (SvA 19, 576)

Khokhlova V.L., Pavlova V.M., 1984, Pis'ma AZh 10, 377 (SvA Lett. 10, 158)

Kron G.E., 1952, ApJ 115, 301

Landstreet J.D., 1988, ApJ 326, 967

Lanz T., 1987, PhD thesis, Observatoire de Genève

Mathys G., 1991, A\&AS 89, 121

Mégessier C., 1975, A\&A 39, 263

Mégessier C., 1984, A\&A 138, 267

Michaud G., 1970, ApJ 160, 641

Michaud G., Mégessier C., Charland Y., 1981, A\&A 103, 244

Neff J.E., Walter F.M., Rodonò M., Linsky J.L., 1989, A\&A 215, 79

Parker E.N., 1970, Ann. Rev. Astron. Astrophys. 8, 1970

Piskunov N.E., 1985, Pis'ma AZh 11, 44 (SvA Lett. 11, 18)

Piskunov N.E., 1991, in: The Sun and Cool Stars: Activity, Magnetism, Dynamos. IAU Coll. 130, 309. Tuominen I., Moss D., Rüdiger G. (eds.). Springer, Berlin Heidelberg New York

Piskunov N.E., Tuominen I., Vilhu O., 1990, A\&A 230, 363

Piskunov N.E., Wehlau W.H., 1990, A\&A 233, 497

Pyper D.M., 1969, ApJS 18, 347

Rice J.B., Wehlau W.H., 1990, A\&A 233, 503

Rice J.B., Wehlau W.H., 1991, A\&A 246, 195

Rice J.B., Wehlau W.H., Khokhlova V.L., 1989, A\&A 208, 179

Rodonò M., Cutispoto G., Pazzani V., et al., 1986, A\&A 165, 135

Saar S.H., 1990, in: Solar Photosphere: Structure, Convection and Magnetic

Fields. Proc. IAU Symp. 138, 427. Stenflo J.O. (ed.). Kluwer, Dordrecht

Semel M., 1989, A\&A 225, 456

Semel M., Donati J.-F., Rees D.E., 1992, A\&A (submitted)

Skilling J., Bryan R.K., 1984, MNRAS 211, 111

Stibbs D.W.N., 1950, MNRAS 110, 395

Stift M.J., Goosens M., 1991, A\&A 251, 139

Strassmeier K.G., Bopp B.W., 1992, A\&A (in press)

Strassmeier K.G., Fekel F.C., Bopp B.W., Dempsey R.C., Henry G.W., 1990, ApJS 72, 191

Strassmeier K.G., Rice J.B., Wehlau W.H., et al., 1991, A\&A 247, 130

Strassmeier K.G., Rice J.B., Wehlau W.H., Hill G., Matthews J.M., 1992, A\&A (submitted)

Tikhonov A.N., 1963, Sov. Math. Dokl. 4, 1624

Toner C.G., Gray D.F., 1988, ApJ, 334, 1008

Vauclair S., Hardorp J., Peterson D.M., 1979, ApJ 227, 526

Vogt S.S., 1981, ApJ 250, 327

Vogt S.S., 1988, in: The Impact of Very High S/N Spectroscopy on Stellar Physics. IAU. Symp. 132, 253. Cayrel de Strobel G., Spite M. (eds.). Kluwer, Dordrecht

Vogt S.S., Hatzes A.P., 1991, in: The Sun and Cool Stars: Activity, Magnetism, Dynamos. IAU Coll. 130, 297. Tuominen I., Moss D., Rüdiger G. (eds.). Springer, Berlin Heidelberg New York

Vogt S.S., Penrod G.D., 1983, PASP 95, 565

Vogt S.S., Penrod G.D., Hatzes A.P., 1987, ApJ 321, 496

Zahn J.-P., 1977, A\&A 57, 383 